\title{
Self-reported confidence in patient safety competencies among Chinese nursing students: a multi-site cross-sectional survey
}

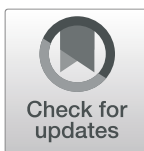

\author{
Fei Fei Huang ${ }^{1 *}$, Xiao Ying Shen ${ }^{2}$, Xue Lei Chen ${ }^{3}$, Li Ping He ${ }^{4}$, Su Fen Huang ${ }^{5}$ and Jin Xiu Li ${ }^{6}$
}

\begin{abstract}
Background: Nursing interns are an important backup force for nursing professionals, so efforts to strengthen their patient safety (PS) competencies are a major priority. To do so requires assessing the strengths and weaknesses of Chinese nursing students' PS competence and identifying the influencing factors.

Methods: This was a multi-site, cross-sectional, web-based study that was carried out between September 2018 and January 2019. A national online survey was completed by 732 Chinese undergraduate nursing students. Our primary outcome factor was the Health Professional Education in Patient Safety Survey score. We also collected socio-demographic and clinical practice-related characteristics as independent variables. Multiple stepwise linear regression was performed to identify predictors of PS competence.

Results: Chinese undergraduate nursing students were fairly confident in their clinical safety skills but less confident in what they learned about sociocultural or context-dependent aspects of PS and speaking up about PS, including effective communication and understanding human and environmental factors. Less than half of the students felt that they could approach someone engaging in unsafe practice and were reluctant to voice concern about adverse events. We observed significant differences in PS competence between students from different regions, across different PS learning styles (self-study and classroom theoretical study), with different self-assessed PS competence levels, and with experiences of adverse events $(p<0.05)$. These factors accounted for almost 15\% of the total variance in PS competence scores (adjusted $R^{2}=0.15, p=0.00$ ).

Conclusions: The results of this study provide a better understanding of PS competence among final-year nursing students in China. Our findings may help nursing educators or healthcare organizations to cultivate and improve PS competence by establishing documented policies or by improving the efficacy of intervention.
\end{abstract}

Keywords: Patient safety, Nursing, Survey, Undergraduate students

\section{Background}

Patient safety (PS) remains under constant scrutiny due to the possibility of preventable harm occurring during medical care [1]. In Chinese hospitals, $40 \%$ of patients experience at least one preventable adverse event; this is worrying because such events can increase the risk of mortality and damage of hospital reputation [2]. It is therefore important that

\footnotetext{
*Correspondence: pt860315@163.com

'School of Nursing, Fujian Medical University, No 1 Xuefu north Road,

Minhou county, Fuzhou 350108, Fujian, China

Full list of author information is available at the end of the article
}

healthcare providers are sufficiently knowledgeable to recognize potential safety risks and protect patients from potential harm or avoidable injuries [3]. Nurses represent the front line of care and the largest group of healthcare providers; consequently, they play a vital role in improving the safety and quality of patient care [4].

Nursing students serve as the important reserve force of healthcare providers. Emphasis that efforts to help nursing students reflect on their PS knowledge and competence may prepare them to offer appropriate and safe

(c) The Author(s). 2020 Open Access This article is distributed under the terms of the Creative Commons Attribution 4.0 International License (http://creativecommons.org/licenses/by/4.0/), which permits unrestricted use, distribution, and reproduction in any medium, provided you give appropriate credit to the original author(s) and the source, provide a link to the Creative Commons license, and indicate if changes were made. The Creative Commons Public Domain Dedication waiver (http://creativecommons.org/publicdomain/zero/1.0/) applies to the data made available in this article, unless otherwise stated. 
care in a variety of clinical settings and circumstances [5]. The first step towards this goal is assessing the strengths and weaknesses of nursing students' PS competence $[3,6]$. The PS competence entails six domains: contributing to patient safety culture, working in teams, communicating effectively, managing safety risks, optimizing human and environmental factors, and recognizing and responding to adverse events. Previous studies have explored the PS knowledge and skills of students such as in nursing, pharmacy, and traditional medicine $[1,3,6$,$] and new graduate health-$ care professionals [7-9] but limited information is available for final-year nursing students or interns, who are the most likely to commit medical errors [1]. It is estimated that 17 to $53.2 \%$ of Chinese nursing interns directly or indirectly cause one or more adverse events $[10,11]$. In previous studies, nursing students have reported moderate levels of confidence in most domains of PS competence [3, 6, 12]. However, the evidence for PS competence varies according to the academic year of study, some publications report that confidence in PS competence declines as students are increasingly exposed to the clinical environment [3, 12], while others reported findings that directly opposed to those opinion [13]. More studies are therefore needed to better understand the PS competence of this vulnerable body of nursing students.

Notably, the PS competence acquired by nursing students in the classroom is lower than that gained in the clinical setting $[3,8]$. For example, undergraduate nursing students in Australia reported greater confidence in their clinical safety skills as opposed to their skills learned in the classroom, although their confidence regarding teamwork skills and managing safety risks was reduced in the clinical setting [3]. The specific domains of PS competence showing discrepancies between the classroom and clinic are heterogeneous when compared across different countries; this may be due to differences in PS cultures [8]. However, the existing evidence was mostly collected in developed countries [3, 8], with more limited datasets from developing countries (e.g., Korea, Saudi Arabia and Jordan) [1, 13-15]. To our knowledge, little is known about the development of nursing students' PS knowledge and confidence in China, where PS strategies and education tend to lag behind those in developed countries [16].

There is increasing evidence that more emphasis is being placed on explicit PS competence for undergraduate nursing education and learning [3]. Age, gender, region, academic year, and employment status were previously found to be associated with the PS competence of nursing students $[9,13]$. However, these findings were obtained using univariate inferential statistical analyses
[15]; confirmatory regression analysis is still required. In addition, knowledge and experience are known to underpin PS competence [7]. Hospital rank, ${ }^{1}$ educational background, experiences of adverse events and reporting behavior may also influence the PS competence of nursing students.

The purposes of this nationwide cross-sectional study were to: (a) describe and compare Chinese undergraduate nursing students' perception of confidence in PS knowledge and competence acquired in both classroom and clinical settings, (b) describe nursing students' confidence relating to broader aspects of PS and speaking up about PS, and (c) explore the factors that might influence nursing students' PS competence.

\section{Methods \\ Study design}

This was a multi-site, cross-sectional, web-based study that was performed between September 2018 and January 2019. The study conformed with the Strengthening the Reporting of Observational studies in Epidemiology (STROBE) statement [17].

\section{Setting and participants}

Seven four-year nursing Bachelor Program of Chinese universities were invited to participate as research partners. To obtain a representative sample, the universities were selected from seven Chinese administrative provincial regions and were representative of the population density, economic development, and medical services of their respective regions. The seven provincial regions were as follows: North (Shanxi), East (Fujian), Northeast (Heilongjiang), Central (Hunan), Southwest (Guizhou), South (Hainan), and Northwest (Xinjiang) China. In addition, one to two nursing faculty members at each participating university agreed to be a research partner and act as a point of contact at their university. Undergraduate nursing students at each of the seven participating universities who were (a) undertaking final-year clinical internship practice and (b) willing to participate in this study were eligible. According to Pett et al. (2003) [18], 10-15 participants per item were considered appropriate for a target sample size. Considering a potential non-response rate of $15 \%$, the final target sample size was 770 . That is, at each university, 110 eligible participants were recruited by convenience sampling methods.

\footnotetext{
${ }^{1}$ Hospitals in China are organized according to a 3 -tier system that recognizes a hospital's ability to provide medical care, medical education, and conduct medical research. Based on this, hospitals are designated as Primary, Secondary or Tertiary institutions.
} 


\section{Measures}

The main outcome factor in our study was the Health Professional Education in Patient Safety Survey (HPEPSS), which was used to measure the self-reported PS competence of health professionals and students [19]. The H-PEPSS has been translated into Chinese and its reliability and validity have been confirmed [20]. The Chinese version of H-PEPSS (H-PEPSS-CV) consists of 32 items divided into three sections, as follows.

Section 1: "Students' knowledge about specific patient safety content areas." This section features two versions of classroom and clinical practice. Each version includes 20 items covering clinical safety issues (4 items) and six dimensions of the Safety Competencies Framework (Ginsburg et al., 2012): communicating effectively (3 items); working in a team with other health professionals (3 items); managing safety risks (3 items); understanding human and environmental factors ( 2 items); recognizing, responding to, and disclosing adverse events and close calls (2 items); and the culture of safety (3 items).

Section 2: "How broader patient safety issues are addressed in health professional education." (7 items). Section 3: "How able and comfortable the participants are speaking up about patient safety issues." (5 items).

Each section is completed by adding together the students' responses to each item. For item scoring, responses range from "strongly disagree" (scored as 1) to "strongly agree" (scored as 5). Higher domain scores or total scores represent higher levels of students' perceived PS competence. The scale has achieved a Cronbach's $\alpha$ of 0.95 and 2-week test-retest reliability of 0.88 .

The socio-demographic and clinical practice-related characteristics as independent variables were also collected, including age, gender, only-child status, hospital rank, whether the participant had previously received PS education, whether the participant had experienced adverse events, whether they had disclosed medical errors, and their PS learning method (self-study, theoretical study in the classroom or clinical training).

\section{Data collection}

An online questionnaire (hosted by Wenjuanxing, http:// www.wjx.cn, a popular online survey platform in China) was made available to all eligible nursing students at each participating university. Before survey, all participants were obtained by written informed consent. To encourage participation, all nursing students at these seven universities were told that this web-based survey was voluntary, anonymous, and confidential, and also that $80 \%$ of them would randomly receive a bonus (named as "Hongbao" in Chinese) (containing 10 yuan) after they completed the questionnaire.

\section{Data analysis}

SPSS 24.0 software (SPSS Inc., Chicago, IL) was used to perform descriptive and inferential statistical analyses. Missing data were replaced using mean value substitution, and $p<0.05$ was considered to be statistically significant. Socio-demographic and clinical practice-related data were summarized using descriptive statistics. Mean ( \pm standard deviation) PS scores for each domain were calculated by averaging the items. Paired t-tests were performed to identify significant differences between classroom and clinical scores. Cohen's effect size was calculated for statistically significant pairwise comparisons.

Scores relating to broader aspects of PS and speaking up were categorized into strongly agree/agree (4$5)$, and neutral/disagree/strongly disagree (1-3); the proportion of students who strongly agreed/agreed were reported descriptively. Chi square tests were conducted using categorized outcomes. Independent t-tests and one-way analyses of variance (ANOVAs) were performed to identify differences in PS competence scores across the independent variables. Multiple stepwise linear regression was performed to identify predictors of PS competence; the dependent variable was PS competence score. Variables showing statistical significance in the t-test or one-way ANOVA were then selected as independent variables for subsequent analysis $(p<0.05)$. Categorical variables were recoded into dummy variables for the multiple linear regression analysis.

\section{Results}

A total of 732 valid questionnaires were returned out of the 770 that were distributed (representing a $95.06 \%$ response rate). The mean age of students was $21.56 \pm 0.96$ years. Of the entire cohort, $97.70 \%$ of the students were undertaking clinical practice in a grade A tertiary hospital, and 1.10 and $0.50 \%$ of the students were working in grade A secondary and grade B tertiary hospitals, respectively. Socio-demographic and educational characteristics of the nursing students involved in this study are shown in Table 1.

\section{Confidence in PS dimensions in classroom and clinical settings}

As shown in Table 2, the mean scores were all above 3.5 (out of 5) for PS dimensions and individual items in the classroom and clinical settings. At the dimension level, nursing students were most confident in their learning of "clinical safety skills" in the classroom $(4.1 \pm 0.58)$ and "managing safety risks" in the 
Table 1 Socio-demographic characteristics of participants $(n=732)$

\begin{tabular}{|c|c|c|c|c|c|c|c|c|c|}
\hline \multirow[t]{2}{*}{ Variable } & \multirow[t]{2}{*}{ n (\%) } & \multicolumn{2}{|c|}{ In the classroom } & \multicolumn{2}{|c|}{ In the clinical practice } & \multicolumn{2}{|c|}{$\begin{array}{l}\text { Broader aspects of patient } \\
\text { safety }\end{array}$} & \multicolumn{2}{|c|}{$\begin{array}{l}\text { Comfort in speaking up about } \\
\text { patient safety. }\end{array}$} \\
\hline & & Total scores & $t / F$ value & Total scores & $t / F$ value & Total scores & $t / F$ value & Total scores & $t / F$ value \\
\hline \multicolumn{10}{|l|}{ Gender } \\
\hline Male & $60(8.20)$ & $78.62 \pm 12.69$ & -0.34 & $78.42 \pm 14.08$ & -0.07 & $26.52 \pm 4.97$ & -0.10 & $17.58 \pm 3.50$ & 0.11 \\
\hline Female & $672(91.80)$ & $79.11 \pm 10.43$ & & $78.52 \pm 10.44$ & & $26.57 \pm 4.33$ & & $17.54 \pm 3.09$ & \\
\hline \multicolumn{10}{|l|}{ The only-child } \\
\hline Yes & 143(19.60) & $81.75 \pm 10.63$ & $3.39^{*}$ & $81.47 \pm 11.36$ & $3.70^{*}$ & $27.50 \pm 4.57$ & $2.73^{*}$ & $17.84 \pm 3.12$ & 1.28 \\
\hline No & $589(80.40)$ & $78.41 \pm 10.53$ & & $77.79 \pm 10.52$ & & $26.34 \pm 4.31$ & & $17.47 \pm 3.12$ & \\
\hline \multicolumn{10}{|c|}{ Prior experience of patient safety education } \\
\hline Yes & $583(79.70)$ & $80.14 \pm 10.25$ & $5.52^{*}$ & $79.50 \pm 10.41$ & $4.99^{*}$ & $26.95 \pm 4.17$ & $4.76^{*}$ & $17.54 \pm 3.18$ & -0.03 \\
\hline No & 149(20.30) & $74.85 \pm 11.07$ & & $74.62 \pm 11.33$ & & $25.06 \pm 4.87$ & & $17.55 \pm 2.92$ & \\
\hline \multicolumn{10}{|c|}{ The safety learning methods } \\
\hline \multicolumn{10}{|l|}{ Self-study } \\
\hline No & $561(76.60)$ & $78.23 \pm 10.67$ & $-3.90^{*}$ & $77.76 \pm 10.85$ & $-3.43^{*}$ & $26.24 \pm 4.34$ & $-3.75^{*}$ & $17.39 \pm 3.02$ & $-2.32^{*}$ \\
\hline Yes & $171(23.40)$ & $81.81 \pm 10.04$ & & $80.96 \pm 10.20$ & & $27.66 \pm 4.37$ & & $18.02 \pm 3.42$ & \\
\hline \multicolumn{10}{|c|}{ Theoretical study in Classroom } \\
\hline Yes & $526(71.90)$ & $76.24 \pm 10.55$ & $-4.55^{*}$ & $75.76 \pm 10.83$ & $-4.36^{*}$ & $25.40 \pm 4.82$ & $-4.24^{*}$ & $17.45 \pm 3.13$ & -.465 \\
\hline No & $206(28.10)$ & $80.17 \pm 10.46$ & & $79.58 \pm 10.57$ & & $27.02 \pm 4.12$ & & $17.57 \pm 3.12$ & \\
\hline \multicolumn{10}{|l|}{ Clinical training } \\
\hline Yes & $470(64.20)$ & $77.12 \pm 10.83$ & $-3.72^{*}$ & $76.88 \pm 11.13$ & $-3.06^{*}$ & $25.74 \pm 4.84$ & $-3.85^{*}$ & $17.36 \pm 3.09$ & -1.13 \\
\hline No & 262(35.80) & $80.15 \pm 10.37$ & & $79.41 \pm 10.48$ & & $27.03 \pm 4.04$ & & $17.64 \pm 3.14$ & \\
\hline \multicolumn{10}{|c|}{ Experience of adverse events } \\
\hline Yes & 156(21.30) & $76.90 \pm 10.96$ & $-2.95^{*}$ & $76.52 \pm 11.12$ & $-2.68^{*}$ & $25.75 \pm 4.23$ & $-2.88^{*}$ & $17.55 \pm 2.81$ & -0.15 \\
\hline No & $576(78.70)$ & $79.72 \pm 10.38$ & & $79.11 \pm 10.55$ & & $26.85 \pm 4.25$ & & $17.59 \pm 3.12$ & \\
\hline \multicolumn{10}{|c|}{ Disclosing behavior } \\
\hline Yes & $690(94.30)$ & $79.64 \pm 10.48$ & $2.12^{*}$ & $79.06 \pm 10.66$ & 1.64 & $26.90 \pm 4.10$ & 1.25 & $17.65 \pm 3.02$ & -0.39 \\
\hline No & $42(5.60)$ & $75.74 \pm 12.24$ & & $75.98 \pm 12.41$ & & $25.74 \pm 5.40$ & & $17.86 \pm 3.19$ & \\
\hline \multicolumn{10}{|c|}{ Self-assessment patient safety competence } \\
\hline Very well & $66(9.00)$ & $81.71 \pm 13.32$ & $14.96^{*}$ & $81.60 \pm 13.60$ & $13.20^{*}$ & $27.95 \pm 5.61$ & $8.43^{*}$ & $18.31 \pm 3.84$ & 1.13 \\
\hline Well & 293(40.00) & $81.96 \pm 10.23$ & & $81.26 \pm 10.49$ & & $27.51 \pm 4.43$ & & $17.49 \pm 3.54$ & \\
\hline moderate & $348(47.50)$ & $76.77 \pm 9.21$ & & $76.22 \pm 9.56$ & & $25.64 \pm 3.71$ & & $17.41 \pm 2.61$ & \\
\hline Poor & $21(2.90)$ & $73.10 \pm 11.48$ & & $72.71 \pm 8.91$ & & $25.24 \pm 4.39$ & & $17.86 \pm 2.29$ & \\
\hline Very poor & $4(0.50)$ & $55.50 \pm 16.34$ & & $58.25 \pm 12.82$ & & $23.00 \pm 11.05$ & & $18.25 \pm 2.36$ & \\
\hline \multicolumn{10}{|l|}{ Regions } \\
\hline Fujian & $161(22.00)$ & $76.03 \pm 10.89$ & $5.80^{*}$ & $75.33 \pm 11.14$ & $7.25^{*}$ & $24.61 \pm 4.96$ & $11.96^{*}$ & $16.60 \pm 3.02$ & $3.96^{*}$ \\
\hline Guangxi & $76(10.40)$ & $76.84 \pm 9.01$ & & $74.93 \pm 8.71$ & & $25.26 \pm 3.39$ & & $17.49 \pm 2.79$ & \\
\hline Guizhou & $89(12.20)$ & $78.66 \pm 9.29$ & & $78.20 \pm 9.72$ & & $26.98 \pm 3.99$ & & $18.01 \pm 3.27$ & \\
\hline Heilongjian & $125(17.10)$ & $82.02 \pm 11.29$ & & $82.06 \pm 11.07$ & & $28.26 \pm 4.32$ & & $18.07 \pm 3.32$ & \\
\hline Hunan & $114(15.60)$ & $80.57 \pm 9.23$ & & $80.30 \pm 9.11$ & & $27.04 \pm 3.39$ & & $17.79 \pm 2.51$ & \\
\hline Shanxi & $106(14.50)$ & $78.80 \pm 9.41$ & & $78.93 \pm 10.10$ & & $26.87 \pm 3.96$ & & $17.39 \pm 2.82$ & \\
\hline Xinjiang & $61(8.40)$ & $82.14 \pm 13.86$ & & $80.51 \pm 13.59$ & & $27.95 \pm 4.70$ & & $18.12 \pm 4.13$ & \\
\hline
\end{tabular}


Table 2 Classroom and clinical self-reported patient safety domains scores for undergraduate nursing students $(n=732)$

\begin{tabular}{|c|c|c|c|c|c|c|c|}
\hline \multirow[t]{2}{*}{ Patient safety areas } & \multirow[t]{2}{*}{ Setting } & \multirow{2}{*}{$\begin{array}{l}\text { Mean } \\
\text { (SD) }\end{array}$} & \multirow{2}{*}{$\begin{array}{l}\text { Effect } \\
\text { size }\end{array}$} & \multirow{2}{*}{$\begin{array}{l}\text { Paired } t \text {-test ( } t \\
\text { and } p \text { value) }\end{array}$} & \multicolumn{3}{|c|}{ Agree/strongly agree } \\
\hline & & & & & $\mathrm{N}$ & $\%$ & $x^{2} 2$-tail $p$ value \\
\hline \multirow[t]{2}{*}{ Culture of safety } & Classroom & $3.91(0.65)$ & -0.02 & $t=-0.96 p=0.34$ & 558 & 76.17 & $x^{2}=0.19 p=0.66$ \\
\hline & Clinical & $3.94(0.68)$ & & & 565 & 77.17 & \\
\hline \multirow[t]{2}{*}{ Work in teams with other health professionals } & Classroom & $4.1(0.60)$ & 0.14 & $t=9.34 p=0.00$ & 618 & 84.47 & $x^{2}=13.70 p=0.00$ \\
\hline & Clinical & $3.93(0.61)$ & & & 562 & 76.8 & \\
\hline \multirow[t]{2}{*}{ Communicating effectively } & Classroom & $3.92(0.66)$ & 0.11 & $t=7.41 p=0.00$ & 549 & 74.93 & $x^{2}=10.44 p=0.00$ \\
\hline & Clinical & $3.77(0.65)$ & & & 493 & 67.40 & \\
\hline \multirow[t]{2}{*}{ Managing safety risks } & Classroom & $3.82(0.67)$ & -0.14 & $t=-8.54 p=0.00$ & 508 & 69.40 & $x^{2}=28.53 p=0.00$ \\
\hline & Clinical & $4.00(0.59)$ & & & 596 & 81.43 & \\
\hline \multirow[t]{2}{*}{ Understanding human \& environmental factors } & Classroom & $3.79(0.70)$ & -0.10 & $t=-5.95 p=0.00$ & 500 & 68.25 & $x^{2}=15.03 p=0.00$ \\
\hline & Clinical & $3.92(0.63)$ & & & 566 & 77.3 & \\
\hline \multirow[t]{2}{*}{ Recognize \& respond to remove immediate risks } & Classroom & $3.90(0.66)$ & -0.02 & $t=-1.19 p=0.23$ & 558 & 76.20 & $x^{2}=1.42 p=0.43$ \\
\hline & Clinical & $3.93(0.63)$ & & & 577 & 78.80 & \\
\hline \multirow[t]{2}{*}{ Clinical safety skills } & Classroom & $4.11(0.58)$ & 0.11 & $t=6.84 p=0.00$ & 612 & 83.63 & $x^{2}=2.66 p=0.06$ \\
\hline & Clinical & $3.98(0.60)$ & & & 588 & 80.33 & \\
\hline \multirow[t]{2}{*}{ Total score } & Classroom & $4.94(0.66)$ & 0.02 & $t=2.61 p=0.00$ & 557 & 76.15 & $x^{2}=0.19 p=0.66$ \\
\hline & Clinical & $4.91(0.67)$ & & & 564 & 77.03 & \\
\hline
\end{tabular}

clinical setting $(4.00 \pm 0.59)$. They were least confident in their learning of "understanding human and environmental factors" in the classroom $(3.79 \pm 0.70)$ and "communicating effectively" in the clinical setting $(3.77 \pm 0.65)$. The proportion of nursing students who were confident about what they were learning with regards to each PS dimension (based on responding "agree" or "strongly agree") ranged from 68.25 to $84.47 \%$ for classroom learning, and from 67.40 to $81.43 \%$ for learning in the clinical setting.

There are significantly statistical difference between classroom learning and learning in the clinical setting on mean scores of the majority of PS dimensions, such as "clinical safety skills," "working in teams with other health professionals," and "communicating effectively" (all $p<0.05$ ). All of these statistically significant differences in PS dimensions had small effect sizes and are therefore likely to be of low clinical significance. There were no significant differences in the remaining PS dimensions, such as the culture of safety.

The trend for different learning confidence levels between the classroom and clinical setting was further explored by examining specific items. Except for the "recognizing an adverse event or close call" item, we found statistically significant differences (all with small effect sizes) for all the PS items between the classroom and clinical setting. Furthermore, we identified significant correlations between the students' scores for the classroom and clinical setting $(p=0.00)$ for most items (Table 3).

\section{Confidence in knowledge relating to "broader aspects of PS" and "comfort with speaking up"}

Nursing students' self-reported confidence in their learning of "broader aspects of PS" and "comfort with speaking up" was generally lower than their confidence in the seven items related to the "broader aspects of PS" (Table 4). The nursing students most strongly agreed that "reporting can lead to change and improvement" (84.50\%),"clinical aspects (e.g., hand hygiene, transferring patients, and medication safety) were well covered in the program" (83.4\%), and their "scope of practice is clear" (73.7\%). While, other areas pertaining to broader aspects of PS and comfort with speaking up about PS issues had agreement levels below 50\%, including "felt they could approach someone engaging in unsafe practice" (44.80\%), "consistency in how PS issues are dealt with by different instructors" (46.90\%), "it is difficult to question the decisions or actions of those with more authority" (47.00\%), and "reporting PS problems will result in negative repercussions for them" (48.80\%).

\section{Factors that influence nursing students' perceived confidence relating to PS dimensions}

Table 1 shows significant differences for various independent variables in confidence relating to PS dimensions learned in the classroom and clinical settings, knowledge of broader aspects of PS, and comfort with speaking up. These independent variables were included in the four corresponding regression models.

Table 5 highlights the four factors that could predict PS competence among Chinese undergraduate nursing 
Table 3 Descriptive statistics of each H-PEPSS-CV item $(n=732)$

\begin{tabular}{|c|c|c|c|c|c|c|c|}
\hline \multirow[t]{2}{*}{ Patient safety areas } & \multirow[t]{2}{*}{ Setting } & \multirow{2}{*}{$\begin{array}{l}\text { Mean } \\
(\mathrm{SD})\end{array}$} & \multirow{2}{*}{$\begin{array}{l}\text { Effect } \\
\text { size }\end{array}$} & \multirow{2}{*}{$\begin{array}{l}\text { Paired } t \text {-test } \\
\text { ( } t \text { and } p \text { value) }\end{array}$} & \multicolumn{3}{|c|}{ Agree/strongly agree } \\
\hline & & & & & $\overline{\mathrm{N}}$ & $\%$ & $x^{2} 2$-tail $p$ value \\
\hline \multirow[t]{2}{*}{ T1 Safe clinical practice in general } & Classroom & $3.76(0.82)$ & -0.07 & $t=-3.79$ & 510 & 69.70 & $x^{2}=8.68$ \\
\hline & Clinical & $3.87(0.77)$ & & $p=0.00$ & 560 & 76.50 & $p=0.00$ \\
\hline \multirow[t]{2}{*}{ T2 Hand hygiene } & Classroom & $4.00(0.68)$ & -0.07 & $t=-3.79$ & 607 & 82.90 & $x^{2}=0.99$ \\
\hline & Clinical & $4.10(0.70)$ & & $p=0.00$ & 621 & 84.90 & $p=0.32$ \\
\hline \multirow[t]{2}{*}{ T3 Infection control } & Classroom & $4.28(0.81)$ & 0.27 & $t=14.49$ & 657 & 89.80 & $x^{2}=57.45$ \\
\hline & Clinical & $3.84(0.78)$ & & $p=0.00$ & 546 & 74.60 & $p=0.00$ \\
\hline \multirow[t]{2}{*}{ T4 Safe medication practices } & Classroom & $4.40(0.69)$ & 0.21 & $t=10.72$ & 674 & 92.10 & $x^{2}=16.99$ \\
\hline & Clinical & $4.10(0.69)$ & & $p=0.00$ & 624 & 85.30 & $p=0.00$ \\
\hline \multirow{2}{*}{$\begin{array}{l}\text { T5 The importance of having a questioning attitude and } \\
\text { speaking up when you see things that may be unsafe }\end{array}$} & Classroom & $3.80(0.80)$ & -0.04 & $t=-2.19$ & 515 & 70.40 & $x^{2}=1.79$ \\
\hline & Clinical & $3.86(0.79)$ & & $p=0.03$ & 538 & 73.50 & $p=0.18$ \\
\hline \multirow{2}{*}{$\begin{array}{l}\text { T6 The importance of a supportive environment that } \\
\text { encourages patients and providers to speak up when } \\
\text { they have safety concerns }\end{array}$} & Classroom & $3.99(0.76)$ & -0.05 & $t=-2.75$ & 580 & 79.30 & $x^{2}=4.04$ \\
\hline & Clinical & $4.07(0.74)$ & & $p=0.01$ & 610 & 83.40 & $p=0.04$ \\
\hline \multirow{2}{*}{$\begin{array}{l}\text { T7 The nature of systems and system failures and their } \\
\text { role in adverse events }\end{array}$} & Classroom & $3.95(0.79)$ & 0.04 & $t=1.90$ & 577 & 78.80 & $x^{2}=3.67$ \\
\hline & Clinical & $3.88(1.06)$ & & $p=0.06$ & 546 & 74.60 & $p=0.06$ \\
\hline \multirow[t]{2}{*}{ T8 Managing inter-professional conflict } & Classroom & $4.18(0.71)$ & 0.09 & $t=5.13$ & 641 & 87.60 & $x^{2}=4.07$ \\
\hline & Clinical & $4.05(0.69)$ & & $p=0.00$ & 614 & 83.90 & $p=0.04$ \\
\hline \multirow[t]{2}{*}{ T9 Sharing authority, leadership and decision-making } & Classroom & $3.95(0.78)$ & 0.11 & $t=6.12$ & 572 & 78.20 & $x^{2}=14.46$ \\
\hline & Clinical & $3.78(0.78)$ & & $p=0.00$ & 508 & 69.40 & $p=0.00$ \\
\hline \multirow{2}{*}{$\begin{array}{l}\text { T10 Encouraging team members to speak up, question, } \\
\text { challenge, advocate, and be accountable as appropriate } \\
\text { to address safety issues }\end{array}$} & Classroom & $4.18(0.70)$ & 0.16 & $t=9.09$ & 641 & 87.60 & $x^{2}=27.81$ \\
\hline & Clinical & $3.95(0.73)$ & & $p=0.00$ & 564 & 77.10 & $p=0.00$ \\
\hline \multirow{2}{*}{$\begin{array}{l}\text { T11 Enhancing patient safety through clear and consistent } \\
\text { communication with patients }\end{array}$} & Classroom & $3.88(0.82)$ & 0.15 & $t=8.85$ & 547 & 74.70 & $x^{2}=40.11$ \\
\hline & Clinical & $3.63(0.82)$ & & $p=0.00$ & 433 & 59.10 & $p=0.00$ \\
\hline \multirow{2}{*}{$\begin{array}{l}\text { T12 Enhancing patient safety through effective } \\
\text { communication with other healthcare providers }\end{array}$} & Classroom & $4.07(0.76)$ & 0.19 & $t=10.11$ & 596 & 81.40 & $x^{2}=50.19$ \\
\hline & Clinical & $3.77(0.80)$ & & $p=0.00$ & 476 & 65.00 & $p=0.00$ \\
\hline \multirow{2}{*}{$\begin{array}{l}\text { T13 Effective verbal and nonverbal communication } \\
\text { abilities to prevent adverse events }\end{array}$} & Classroom & $3.80(0.82)$ & -0.06 & $t=-3.62$ & 503 & 68.70 & $x 2=16.69$ \\
\hline & Clinical & $3.90(0.73)$ & & $p=0.00$ & 572 & 78.10 & $p=0.00$ \\
\hline \multirow{2}{*}{$\begin{array}{l}\text { T14 Recognizing routine situations in which safety } \\
\text { problems may arise }\end{array}$} & Classroom & $3.98(0.75)$ & -0.08 & $t=-4.23$ & 564 & 77.10 & $x^{2}=17.87$ \\
\hline & Clinical & $4.10(0.70)$ & & $p=0.00$ & 627 & 85.60 & $p=0.00$ \\
\hline \multirow[t]{2}{*}{ T15 Identifying and implementing safety solutions } & Classroom & $3.68(0.81)$ & -0.12 & $t=-6.33$ & 463 & 63.30 & $x 2=24.85$ \\
\hline & Clinical & $3.86(0.74)$ & & $p=0.00$ & 551 & 75.30 & $p=0.00$ \\
\hline \multirow[t]{2}{*}{ T16 Anticipating and managing high risk situations } & Classroom & $3.80(0.79)$ & -0.17 & $t=-8.81$ & 496 & 67.80 & $x^{2}=48.05$ \\
\hline & Clinical & 4.05(0.69) & & $p=0.00$ & 610 & 83.40 & $p=0.00$ \\
\hline \multirow{2}{*}{$\begin{array}{l}\text { T17 The role of human factors, such as fatigue, which } \\
\text { effect patient safety }\end{array}$} & Classroom & $3.70(0.81)$ & -0.07 & $t=-3.86$ & 465 & 63.50 & $x 2=12.84$ \\
\hline & Clinical & $3.81(0.77)$ & & $p=0.00$ & 529 & 72.30 & $p=0.00$ \\
\hline \multirow{2}{*}{$\begin{array}{l}\text { T18 The role of environmental factors such as work } \\
\text { flow, ergonomics and resources, which effect patient safety }\end{array}$} & Classroom & $3.86(0.76)$ & -0.12 & $t=-6.38$ & 534 & 73.0 & $x^{2}=18.19$ \\
\hline & Clinical & $4.03(0.69)$ & & $p=0.00$ & 602 & 82.30 & $p=0.00$ \\
\hline T19 Recognizing an adverse event or close call & Classroom & $3.84(0.77)$ & 0.01 & $t=0.37$ & 541 & 73.90 & $x 2=0.06$ \\
\hline & Clinical & $3.83(0.75)$ & & $p=0.71$ & 545 & 74.50 & $p=0.81$ \\
\hline T20 Reducing harm by addressing immediate risks for & Classroom & $3.96(0.75)$ & -0.04 & $t=-2.48$ & 575 & 78.50 & $x^{2}=4.80$ \\
\hline & Clinical & $4.02(0.68)$ & & $p=0.01$ & 608 & 83.10 & $p=0.03$ \\
\hline
\end{tabular}


Table 4 Broader aspects of patient safety and comfort in speaking up about patient safety

\begin{tabular}{|c|c|c|c|}
\hline \multirow[t]{2}{*}{ Undergraduate nursing students $(n=732)$} & \multirow[t]{2}{*}{ Mean (SD) } & \multicolumn{2}{|c|}{ Agree/strong agree } \\
\hline & & $\mathrm{N}$ & $\%$ \\
\hline Broader aspects of patient safety & $3.80(0.63)$ & 520 & 71.07 \\
\hline As a student, the scope of what was "safe" for me to do in the practice setting was very clear to me & $3.86(0.77)$ & 539 & 73.70 \\
\hline $\begin{array}{l}\text { There is consistency in how patient safety issues were dealt with by different preceptors in the } \\
\text { clinical setting }\end{array}$ & 3.36(0.97) & 343 & 46.90 \\
\hline I had sufficient opportunity to learn and interact with members of interdisciplinary teams & $3.57(0.94)$ & 452 & 61.80 \\
\hline $\begin{array}{l}\text { I gained a solid understanding that reporting adverse events and close calls can lead to change and can } \\
\text { reduce reoccurrence of events }\end{array}$ & 4.08(0.73) & 619 & 84.50 \\
\hline Patient safety was well integrated into the overall program & 3.80(0.78) & 521 & 71.20 \\
\hline $\begin{array}{l}\text { Clinical aspects of patient safety (e.g. hand hygiene, transferring patients, medication safety] were well } \\
\text { covered in our program }\end{array}$ & $4.02(0.74)$ & 610 & 83.40 \\
\hline "System" aspects of patient safety were well covered in our program & $3.89(0.76)$ & 556 & 76.00 \\
\hline Comfort in speaking up about patient safety & $3.51(0.62)$ & 406 & 55.50 \\
\hline $\begin{array}{l}\text { In clinical settings, discussion around adverse events focuses mainly on system-related issues, rather than } \\
\text { focusing on the individual (s) most responsible for the event }\end{array}$ & $3.70(0.83)$ & 477 & 65.10 \\
\hline $\begin{array}{l}\text { In clinical settings, reporting a patient safety problem will result in negative repercussions for the person } \\
\text { reporting it }\end{array}$ & $3.30(1.03)$ & 357 & 48.80 \\
\hline If I see someone engaging in unsafe care practice in the clinical setting, I feel safe to approach them & 3.45(0.77) & 328 & 44.80 \\
\hline It is difficult to question the decisions or actions of those with more authority & 3.36(0.93) & 344 & 47.00 \\
\hline If I make a serious error, I worry that I will face disciplinary action. & $3.76(0.92)$ & 526 & 71.80 \\
\hline
\end{tabular}

students (relating to three models, i.e., for PS competence based on learning in a classroom and in a clinical setting, and knowledge of the broader aspects of PS): (a) region, (b) PS knowledge gained by self-study, (c) PS knowledge gained by theoretical studies in the classroom, (d) self-assessment of PS competence as "moderate or "poor," and (e) experience of adverse events. These factors accounted for approximately $15 \%$ of the total variance (adjusted $R^{2}=0.15$ ) in PS competence scores. In addition, previous experience of PS education was also a significant influencing factor of PS competence based on learning in a classroom and knowledge of the broader aspects of PS. When investigating students' comfort with speaking up about PS issues, we found that region and selfstudy were statistically significant in the univariate analyses, but neither was significant in the final regression model $(p>0.05)$.

\section{Discussion}

To our knowledge, this is the first study to explore selfreported PS competence and influencing factors among undergraduate nursing students in China. Our results revealed moderate self-reported PS competence among Chinese undergraduate nursing students. This level of competence is higher than for nursing students in Jordan [15], Korea [1] and Canada [12], but slightly lower than the overall PS competence of nursing students in Italy [21], Saudi Arabia [13] and Australia [3]. Our finding that nursing students reported higher levels of PS competence for clinical safety compared to the sociocultural aspects of safety is consistent with previous reports relating to nursing and medical students $[1,3,8]$.

Although the World Health Organization published a multi-professional PS curriculum guide [22], our findings further reflect the limitations of existing nursing education programs for PS. Similar to United Kingdom, Japan and United states, there is currently no formal PS curriculum for undergraduate nursing programs in China [23]. Instead, PS learning is interspersed with other nursing curricula, and the PS contents overwhelmingly focus on clinical safety issues, with insufficient attention paid to the sociocultural aspects of PS [15].

Similar to previous studies [3, 8, 15], we also observed a difference between nursing students' confidence with regards to what they learned in the classroom and in clinical settings. This further highlights the current "theory to practice gap" in PS [3, 8]. Nursing students were more confident about what they learned in the classroom compared with what they learned in clinical practice when considering the dimensions of "clinical safety skills," "communicating effectively about PS issues," and "working in teams with other health professionals." Ways to develop clinical safety knowledge, teamwork, and communication, include increasing the application of approaches for the reform of nursing education, including simulation, team-based learning or problem-based learning. 
Table 5 Independent predictors for the level of confidence in PS competence of nursing students $(n=732)$

\begin{tabular}{|c|c|c|c|c|c|c|}
\hline Section & Predictors & Reference & B & Standardized beta & $t$ & $p$ \\
\hline \multirow{11}{*}{$\begin{array}{l}\text { The seven PS dimensions in the } \\
\text { classroom }^{\text {a }}\end{array}$} & Constant & & 76.62 & & 27.35 & 0.00 \\
\hline & $\begin{array}{l}\text { Had prior experience of patient safety } \\
\text { education }\end{array}$ & No experience & 2.64 & 0.10 & 2.58 & 0.01 \\
\hline & Self-study & Without self-study & 2.35 & 0.09 & 2.64 & 0.01 \\
\hline & Theoretical study in Classroom & Without theoretical study & 1.86 & 0.08 & 2.06 & 0.04 \\
\hline & Had experience of adverse events & No experience & 2.51 & 0.10 & 2.78 & 0.01 \\
\hline & Self-assessment patient safety competence & Very well & & & & \\
\hline & General & & -2.96 & -0.14 & -2.15 & 0.03 \\
\hline & Poor & & -5.95 & -0.09 & -2.36 & 0.02 \\
\hline & Very poor & & -19.87 & -0.14 & -3.87 & 0.00 \\
\hline & Regions & Shanxi & & & & \\
\hline & Xinjiang & & 3.64 & 0.09 & 2.28 & 0.02 \\
\hline \multirow{10}{*}{$\begin{array}{l}\text { The seven PS dimensions in } \\
\text { the clinical setting }{ }^{b}\end{array}$} & Constant & & 74.53 & & 29.45 & 0.00 \\
\hline & Self-assessment patient safety competence & Very well & & & & \\
\hline & General & & -3.77 & -0.18 & -2.69 & 0.01 \\
\hline & Poor & & -7.33 & -0.11 & -2.88 & 0.00 \\
\hline & Very poor & & -18.50 & -0.13 & -3.54 & 0.00 \\
\hline & Regions & Shanxi & & & & \\
\hline & Fujian & & -2.98 & -0.12 & -2.36 & 0.01 \\
\hline & Theoretical study in Classroom & Without theoretical study & 2.63 & 0.11 & 3.11 & 0.00 \\
\hline & Had experience of adverse events & No experience & 2.20 & 0.08 & 2.38 & 0.02 \\
\hline & Self-study & Without self-study & 1.93 & 0.08 & 2.12 & 0.04 \\
\hline \multirow[t]{10}{*}{ Broader aspects of PS } & Constant & & 25.80 & & 22.27 & 0.00 \\
\hline & Self-assessment patient safety competence & Very well & & & & \\
\hline & General & & -1.41 & -0.16 & -2.48 & 0.01 \\
\hline & Regions & Shanxi & & & & \\
\hline & Heilongjian & & 1.09 & 0.09 & 1.99 & 0.05 \\
\hline & Fujian & & -2.00 & -0.19 & -3.92 & 0.00 \\
\hline & Theoretical study in Classroom & Without theoretical study & 0.80 & 0.08 & 2.16 & 0.03 \\
\hline & Had experience of adverse events & No experience & 1.12 & 0.10 & 2.99 & 0.00 \\
\hline & $\begin{array}{l}\text { Had prior experience of patient safety } \\
\text { education }\end{array}$ & No experience & 0.88 & 0.08 & 2.08 & 0.04 \\
\hline & Self-study & Without self-study & 0.74 & 0.07 & 2.02 & 0.04 \\
\hline
\end{tabular}

${ }^{\mathrm{a}} \mathrm{F}=10.22, p=0.00$, adjust $R^{2}=0.15$

${ }^{\mathrm{b}} \mathrm{F}=9.74, p=0.00$, adjust $R^{2}=0.14$

${ }^{\mathrm{c}} F=10.09, p=0.00$, adjust $R^{2}=0.15$

However, the confidence of nursing students relating to other intangible dimensions of PS ("managing safety risks" and "understanding human and environmental factors") were higher for clinical practice compared with the classroom. In mainland China, hospitals rather than nursing schools oversee interns' nursing safety problems [11]. This implies that a hospital's culture and clinical practice can promote nursing students' understanding of the human and environmental factors underpinning PS, and their understanding of how to manage safety risks [14, 24].
As nursing students spend more time in the clinic, they gain greater levels of awareness about gaps in their knowledge [6], such as how to communicate effectively and how to work in teams with other health professionals. Fostering collaboration between nursing faculty and clinical nurses to teach PS content could help close the gap between theory and practice in PS education [6]. Improving the overall integration and implementation of PS issues in the classroom and clinical settings are important issues faced by nursing educators and clinical instructors. In other words, we must understand what is 
being taught in both settings. This could help address the inconsistencies in how PS issues are dealt with by different instructors, as reported by undergraduate nursing students (only $46.90 \%$ in our present study). Consistent with previous research [25], this suggests that preceptor consistency is a real unsolved issue across multiple programs in China, which may influence student accountability and confidence in dealing with errors.

Regarding comfort with speaking up about PS issues, our findings are congruent with previous studies and confirm the persistent theme in PS research, that is, the uncertainty and apprehension around error reporting [6]. Being able to raise PS issues comfortably is persistently dependent on the culture and attitudes in each specific clinical setting. "Safety voice" is a form of discretionary communication and is more likely to be used in corporate culture or supportive environments [26]. The nursing field is characterized by hierarchical power dynamics and rigid role boundaries, which make nursing students less likely to raise concerns [3]. This is supported by our finding that $47 \%$ of nursing students comply with unacceptable practices to avoid disrupting their sense of belonging to their nursing team. It is therefore important for nursing educators or clinical instructors to adopt role-modeling behaviors and to guide and encourage nursing students to raise concerns about PS [27]. In addition, they should consider the need for nursing students to freely share and reflect on adverse events/ close calls in a not punitive and more constructive environment and in appropriate settings with modern information technology and mobile devices, such as QQ or WeChat.

Notably, this study also revealed that the perceived PS competence of nursing students varied significantly according to region, self-assessment of PS competence, PS training methods (i.e., self-study and theoretical classroom study) and previous experience of PS education or adverse events. Although the $R^{2}$ values were relatively small, our analyses help to explain the factors that influence PS competence among Chinese undergraduate nursing students; this fills a notable gap in the existing literature. Our findings corroborated those of other multi-center PS studies [1, 3, 13], in that the universities and hospitals where nursing students learn and practice play a key role in influencing the students' competence in PS. On the other hand, it reflects the status quo of nursing education on safety, that is, few nursing schools in mainland China include a nursing safety or PS curriculum in their teaching program, and the undergraduate interns receive their most knowledge of PS from pre-practice education at teaching hospitals.
This underscores the need to establish a strong and coherent PS curriculum. We also gained a better understanding of the barriers to, and facilitators of, PS competence. Specifically, previous experience of adverse events was an important potential barrier to PS competence, while self-study and theoretical learning about PS issues, and having "very good" self-assessment of PS competence were important facilitators. It is necessary to establish effective teaching and learning strategies that equip nursing students with adequate PS-related knowledge and skills to correctly deal with adverse events if we are to achieve meaningful PS improvements and create harm-free environments for patients [28].

This study has several limitations worth noting. Selection bias is a common limitation of cross-sectional studies as probability sampling is seldom used. Additionally, self-reported data (e.g., adverse events or disclosed medical errors) may have introduced a social desirability bias, which could have led to the over- or under-reporting of PS competence. Further investigations, such as qualitative research or a longitudinal single cohort study, are now required to better understand the confidence of nursing students in their classroom and clinical learning.

\section{Conclusions}

Since nursing interns are a vital backup force for nursing professionals, it is critical to strengthen their competence in PS [29]. These results describe the PS competencies of final-year nursing students approaching entry into professional clinical practice. Overall, Chinese nursing students reported moderately high PS competencies, although socio-cultural aspects were weak. Previous experience of adverse events was identified as a barrier to PS competence, while diversified PS training facilitated PS competence. Our findings may help nursing educators and healthcare organizations to better determine how to cultivate and improve PS competence in undergraduate nursing students by establishing documented policies or by improving intervention efficacy.

\section{Abbreviations \\ H-PEPSS: the Health Professional Education in Patient Safety Survey; PS: Patient safety}

\section{Acknowledgements \\ We are grateful to the students who participated in the study and helped improve patient safety education.}

\section{Authors' contributions}

HFF contributed to the conception and design of the work, the interpretation of the data and the drafting of the manuscript. SXY, HSF, and CXL contributed to data collection. LJX and HLP contributed to data analysis and interpretation. All authors approved the final manuscript for publication.

\section{Funding}

The study was funded by the Undergraduate Student Innovation and Entrepreneurship Training Program of Fujian Medical University (grant no. C18155). 


\section{Availability of data and materials}

The datasets used and/or analysed during the current study are available from the corresponding author on reasonable request.

\section{Ethics approval and consent to participate}

Before survey, all participants were obtained by verbal informed consent. The research was approved by the ethical committee of Fujian Medical University (M20170302).

\section{Consent for publication}

Not applicable.

\section{Competing interests}

The authors declare that they have no competing interests.

\section{Author details}

${ }^{1}$ School of Nursing, Fujian Medical University, No 1 Xuefu north Road, Minhou county, Fuzhou 350108, Fujian, China. ${ }^{2}$ School of Nursing, Harbin Medical University, the 2nd Affiliated Hospital of Harbin Medical University, Herbin, China. ${ }^{3}$ School of Nursing, Guilin Medical University, Gulin, China. ${ }^{4}$ School of Nursing, Changzhi Medical College, Changzhi, China. ${ }^{5}$ School of Nursing, Quanzhou Medical College, Quanzhou, China. ${ }^{6}$ School of Nursing, Ji Shou University, Jishou, China.

Received: 5 August 2019 Accepted: 24 January 2020

Published online: 31 January 2020

\section{References}

1. Hwang $\mathrm{J}$, Yoon $T Y$, Jin $\mathrm{HJ}$, et al. Patient safety competence for final-year health professional students: perceptions of effectiveness of an interprofessional education course. J Interprof Care. 2016;30(6):732-8.

2. Zhu H, Liu C, Jin XD. Adverse event reporting system and patient safety. Med and Philos. 2013;34:1-3.

3. Usher K, Woods C, Parmenter G, et al. Self-reported confidence in patient safety knowledge among Australian undergraduate nursing students: a multi-site cross-sectional survey study. Int J Nurs Stud. 2017;71:89-96.

4. Palese A, Gonella S, Grassetti $L$, et al. Multi-level analysis of national nursing students' disclosure of patient safety concerns. Med Educ. 2018; 52(11):1156-66.

5. Kim CH, Jeong SY, Kwon MS. Effects of hazard perception training (HPT) on nursing students' risk sensitivity to patient safety and developing safety control confidence. Appl Nurs Res. 2018;39:160-6.

6. Doyle P, VanDenKerkhof EG, Edge DS, et al. Self-reported patient safety competence among Canadian medical students and postgraduate trainees: a cross-sectional survey. BMJ Qual Saf. 2015;24(2):135-41.

7. Castello M, Ferrara P, Destrebecq A, et al. The perception of clinical risk among students of different health professions: a multicentre study. $\mathrm{Br} J$ Nurs. 2019;28(3):193-7.

8. Ginsburg LR, Tregunno D, Norton PG. Self-reported patient safety competence among new graduate in medicine, nursing and pharmacy. BMJ Qual Saf. 2013:22:147-54.

9. VanDenKerkhof E, Sears N, Edge DS, et al. Patient safety in practical nurses' education: a cross-sectional survey of newly registered practical nurses in Canada. Nurse Educ Today. 2017;51:48-56.

10. Long YF, Li YL, Guo YH. The status quo investigation and analysis of nursing adverse events report in China. Chin Nurs Manage. 2011;11:16-20.

11. Song J, Guo Y. What influences nursing safety event reporting among nursing interns?: focus group study. Nurse Educ Today. 2019;76:200-5.

12. Lukewich J, Edge DS, Tranmer J, et al. Undergraduate baccalaureate nursing students' self-reported confidence in learning about patient safety in the classroom and clinical settings: an annual cross-sectional study (2010-2013). Int J Nurs Stud. 2015:52(5):930-8.

13. Alquwez N, Cruz JP, Alshammari F, et al. A multi-university assessment of patient safety competence during clinical training among baccalaureate nursing students: a cross-sectional study. J Clin Nurs. 2019;28(9-10):1771-81.

14. Colet PC, Cruz JP, Cruz CP, et al. Patient Safety Competence of Nursing Students in Saudi Arabia: A Self-Reported Survey. Int J Health Sci (Qassim). 2015;9(4):418-26.

15. Suliman M. Measuring patient safety competence among nursing students in the classroom and clinical settings. Nurs Educ Perspect. 2019;40(3):E3-7.
16. Liu HP, Wang Q, Liu GY. Development and reflection of nursing safety education in and out of China. Chin Nurs Manage. 2018;18:1316-20.

17. von Elm E, Altman DG, Egger M, et al. STROBE initiative. The strengthening the reporting of observational studies in epidemiology (STROBE) statement: guidelines for reporting observational studies. Int I Surg. 2014;12(12):1495-9.

18. Pett MA, Lackey NR, Sullivan JJ. Making sense of factor analysis: the use of factor analysis for instrument development in health care research. Thousand Oaks, CA: Sage Publications Inc; 2003.

19. Ginsburg LR, Castel E, Tregunno D, et al. The H-PEPSS: an instrument to measure health professionals' perceptions of patient safety competence at entry into practice. BMJ Qual Saf. 2012;21(8):676-84.

20. Chen $L$, Huang F, Yuan $X$, et al. An assessment of the reliability and factorial validity of the Chinese version of the health professional education in patient safety survey (H-PEPSS). Front Psychol. 2019;10:2183. https://doi.org/ 10.3389/fpsyg.2019.02183.

21. Stevanin S, Bressan V, Bulfone G, et al. Knowledge and competence with patient safety as perceived by nursing students: the findings of a crosssectional study. Nurse Educ Today. 2015;35(8):926-34.

22. Mansour M, Skull A, Parker M. Evaluation of World Health Organization multiprofessional patient safety curriculum topics in nursing education: pretest, post-test, none-experimental study. J Prof Nurs. 2015;31 (5):432-9.

23. Ji JX. Investigation and analysis on professional exposure and protection of nursing students in practice. Dissertation. Jinan: Shandong University; 2016.

24. Duhn L, Karp S, Oni O, et al. Perspectives on patient safety among undergraduate nursing students. J Nurs Educ. 2012;51(9):526-31.

25. June MR, Jennifer MM, Christina MG. Baccalaureate nursing students' confidence on patient safety. J Nurs Educ Pract. 2017;7(6):56-64.

26. Dekker SWA, Breakey H. Just culture: improving safety by achieving substantive, procedural and restorative justice. Saf Sci. 2016;85:187-93.

27. Steven A, Magnusson C, Smith P, et al. Patient safety in nursing education: contexts, tensions and feeling safe to learn. Nurse Educ Today. 2014;34(2): 277-84.

28. Fagan A, Parker V, Jackson D. A concept analysis of undergraduate nursing students speaking up for patient safety in the patient care environment. J Adv Nurs. 2016;72(10):2346-57.

29. Li YZ, Wang M, He SH, et al. Study on effects of patient safety education for undergraduate nursing students. Chin J Evidence-based Med. 2014;14(1):30-3.

\section{Publisher's Note}

Springer Nature remains neutral with regard to jurisdictional claims in published maps and institutional affiliations.

Ready to submit your research? Choose BMC and benefit from:

- fast, convenient online submission

- thorough peer review by experienced researchers in your field

- rapid publication on acceptance

- support for research data, including large and complex data types

- gold Open Access which fosters wider collaboration and increased citations

- maximum visibility for your research: over $100 \mathrm{M}$ website views per year

At $\mathrm{BMC}$, research is always in progress.

Learn more biomedcentral.com/submissions 